\title{
Assessing Neuronal Metabolism In Vivo by Modeling Imaging Measures
}

\author{
Olga Ciccarelli, ${ }^{1}$ Ahmed T. Toosy, ${ }^{1}$ Nicola De Stefano, ${ }^{2}$ Claudia A.M. Wheeler-Kingshott, ${ }^{3}$ David H. Miller, ${ }^{3}$ \\ and Alan J. Thompson ${ }^{1}$ \\ ${ }^{1}$ Department of Brain Repair and Rehabilitation, NMR Unit, UCL Institute of Neurology, London WC1N 3BG, United Kingdom, ${ }^{2}$ Department of \\ Neurological and Behavioral Sciences, University of Siena, 53100 Siena, Italy, and ${ }^{3}$ Department of Neuroinflammation, NMR Unit, UCL Institute of \\ Neurology, London, United Kingdom WC1N 3BG
}

Mitochondrial dysfunction contributes to the pathogenesis of many neurological diseases, including multiple sclerosis (MS), but is not directly measurable in vivo. We modeled $N$-acetyl-aspartate (NAA), which reflects axonal structural integrity and mitochondrial metabolism, with imaging measures of axonal structural integrity (axial diffusivity and cord cross-sectional area) to extract its mitochondrial metabolic contribution. Lower residual variance in NAA, reflecting reduced mitochondrial metabolism, was associated with greater clinical disability in MS, independent of structural damage.

\section{Introduction}

Mitochondrial dysfunction in the pathogenesis of multiple sclerosis (MS) is receiving increasing attention (Dutta et al., 2006). Decreased $N$-acetyl-aspartate (NAA) concentration, measured by ${ }^{1} \mathrm{H}-\mathrm{MR}$ spectroscopy (MRS), reflects a combination of both neuronal/axonal loss (Bjartmar et al., 2000) and reduced mitochondrial metabolism (Bates et al., 1996). Isolating the mitochondrial metabolic component of NAA would improve our understanding of disease mechanisms in the CNS.

We propose an indirect estimation of mitochondrial metabolism in vivo by statistically modeling the NAA concentration with imaging measures that reflect structural axonal damage, such as axial diffusivity (derived from diffusion tensor imaging) and cord cross-sectional area (derived from volumetric imaging). In particular, reduced axial diffusivity reflects axonal degeneration (Kim et al., 2006), especially in regions of high fiber coherence, and reduced cord cross-sectional area is found in association with axonal loss (Miller et al., 2002). Thus, we assumed that the structural contributions to NAA were explained by axial diffusivity and cord cross-sectional area; therefore, the residual variance in NAA, when modeling all three variables, contains information specific to neuronal mitochondrial metabolism. We performed this methodology in patients with MS, studied with spinal cord imaging 6 months after a spinal cord relapse. The residual variance in NAA concentration was then used to determine whether

Received June 28, 2010; revised June 28, 2010; accepted July 26, 2010.

This study is supported by the Wellcome Trust Grant 074618/Z/04. The NMR Unit scanner is supported by the MS Society of Great Britain and Northern Ireland. This work was undertaken at UCL Hospitals/UCL, which received a proportion of funding from the Department of Health's National Institute for Health Research Biomedical Research Centres' funding scheme. A.T.T. is supported by the Higher Education Funding Council for England. We thank Dan Altmann for statistical advice.

Correspondence should be addressed to Dr. Olga Ciccarelli, Department of Brain Repair and Rehabilitation, Institute of Neurology, University College London, Queen Square, London WC1N 3BG, UK. E-mail: 0.ciccarelli@ ion.ucl.ac.uk.

DOI:10.1523/JNEUROSCI.3330-10.2010

Copyright $\odot 2010$ the authors $\quad 0270-6474 / 10 / 3015030-04 \$ 15.00 / 0$ the metabolic component of NAA was associated with neurological disability independent of measures of structural damage.

\section{Materials and Methods}

Thirteen MS (McDonald et al., 2001) patients were clinically assessed on the Expanded Disability Status Scale (EDSS) (Kurtzke, 1983), 9-hole peg test (9HPT) (Cutter et al., 1999), timed 25-foot walk test (TWT) (Cutter et al., 1999) and MS Walking Scale-12 (MSWS12) (Hobart et al., 2003) six months after a spinal cord relapse, which was due to a C1-C3 lesion (Table 1). The average of two trials for each hand of the 9HPT and the inverse of the mean of two trials for the TWT were calculated (Fischer et al., 1999). Thirteen age-matched healthy subjects were studied (Table 1). All subjects underwent four spinal cord imaging protocols at 1.5T.

First, we used $\mathrm{T} 2$ and proton density-weighted imaging [repetition time $(\mathrm{TR})=3300 \mathrm{~ms}$, echo time $(\mathrm{TE})=110 \mathrm{~ms}$ and $\mathrm{TR}=3000 \mathrm{~ms}$, $\mathrm{TE}=$ $8.9 \mathrm{~ms}$, respectively; echo train length 33 and 11, respectively; field of view $($ FOV $)=240 \times 240 \mathrm{~mm}^{2}$, matrix $=256 \times 224$, interpolated to $512 \times 512,12$ contiguous sagittal-oblique (Fig. $1 a$ ) and coronal-oblique slices, $3 \mathrm{~mm}$ slice thickness]. One patient, showing swelling of the cord at the level of the lesion, was excluded from analysis. The next analysis was performed with MRS with the proton brain examination point resolved spectroscopy sequence (TE $=30 \mathrm{~ms}, 192$ averages) with chemical shift selective water suppression, cardiac gating $[\mathrm{TR}=3 \mathrm{R}-\mathrm{R}$ intervals $(\sim 3$ s)], and spatial saturation pulses. A single voxel was placed on coronal and sagittal T2 images along the main axis of the cord between $\mathrm{C} 1$ and C3 (Fig. $1 b$ ), as previously described (Ciccarelli et al., 2007). The LCModel was used to calculate the concentration (in $\mathrm{mm}$ ) and the percentage SD of the total NAA, which was retained if the percentage SD was lower than 20 , according to the LCModel manual (version 6.2-0) (Fig. 1e). All spectra acquired met this criterion and were retained.

The third analysis was performed with contiguous zonally magnified orthogonal multislice echo-planar imaging [CO-ZOOM-EPI; TE $=96$ $\mathrm{ms}$, cardiac gating, six nondiffusion weighted (b0), diffusion gradients applied along 60 optimized diffusion-weighted directions, maximum $b$ factor $=1000 \mathrm{~s} / \mathrm{mm}^{2}, \mathrm{FOV}=70 \times 47 \mathrm{~mm}^{2}$ reconstructed to $1 \times 1 \mathrm{~mm}^{2}$, 30 contiguous axial slices, $5 \mathrm{~mm}$ slice thickness] (Dowell et al., 2009). The diffusion tensor was estimated on a voxel-by-voxel basis with the RESTORE (robust estimation of diffusion tensor with outlier rejection) algorithm (Chang et al., 2005), using Camino software (http://web4.cs. 
Table 1. Subjects' clinical characteristics and differences in imaging measures between patients and controls

\begin{tabular}{lccc}
\hline & Patients & Controls & $p$ value \\
\hline Mean age (SD) (years) & $36(8.02)$ & $40.8(12.6)$ & n.s. \\
Gender & 8 women, 5 men & 7 women, 6 men & n.s. \\
Type of MS & 12 RR, 1 SP & $\mathrm{n} / \mathrm{a}$ & \\
Median EDSS (range) & $3.5(1-6.5)$ & $\mathrm{n} / \mathrm{a}$ & \\
Mean NAA (SD) (mm) & $4.2(1.97)$ & $5.9(1.05)$ & $0.034^{*}$ \\
Mean cord cross-sectional area (SD) $\left(\mathrm{mm}^{2}\right)$ & $71.36(7.85)$ & $79.20(6.16)$ & $0.007^{*}$ \\
$\begin{array}{l}\text { Mean axial diffusivity (SD) }\left(\mathrm{mm}^{2} / \mathrm{s} \times 10^{-3}\right) \\
\quad \text { in the spinal cord columns }\end{array}$ & $1.05(0.2)$ & $0.96(0.2)$ & n.s. \\
$\begin{array}{l}\text { Mean axial diffusivity (SD) }\left(\mathrm{mm}^{2} / \mathrm{s} \times 10^{-3}\right) \\
\quad \text { in the entire spinal cord }\end{array}$ & $1.04(0.2)$ & $0.99(0.2)$ & n.s. \\
\hline
\end{tabular}

RR, Relapsing remitting; $\mathrm{SP}=$ secondary progressive; n.s., not significant.

*Mann-Whitney U test.
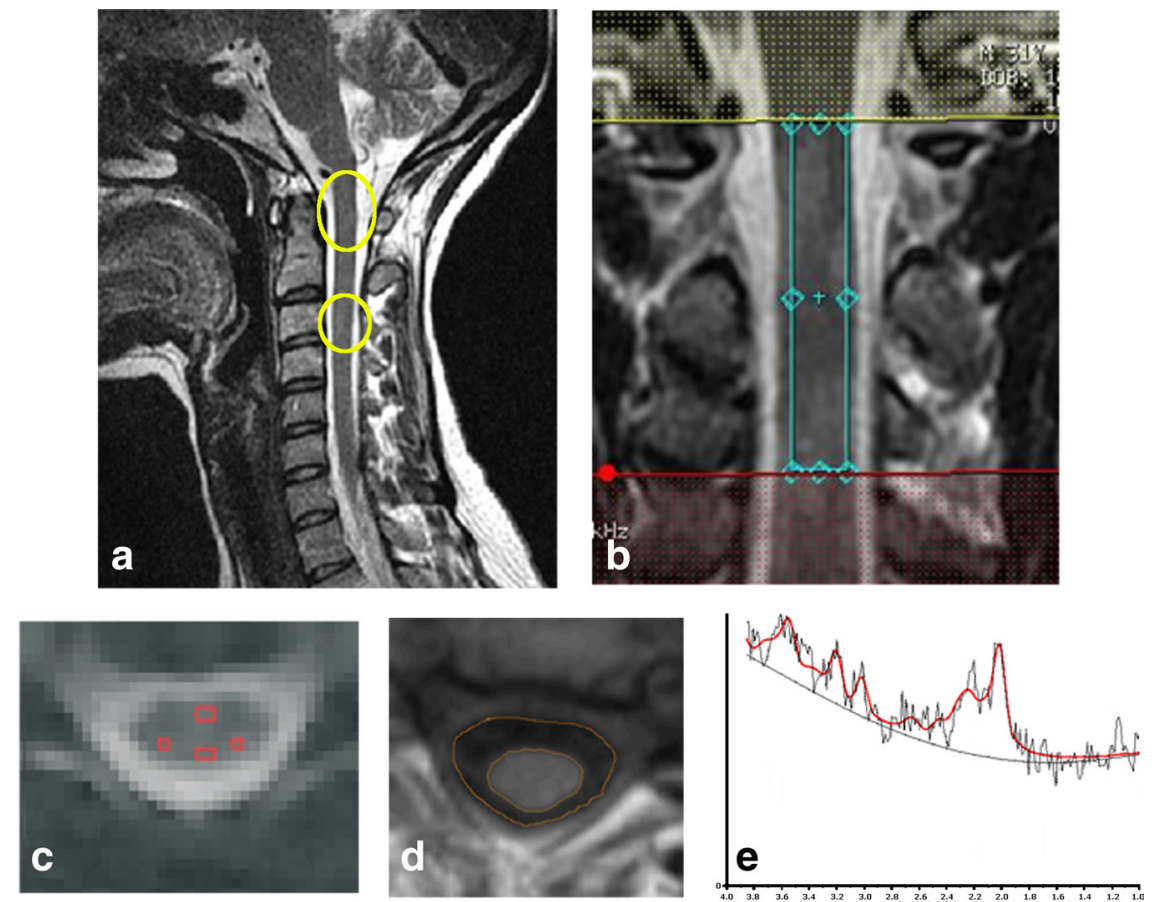

Figure 1. Imaging neuronal metabolism in vivo. $a$, Sagittal 22 image of a patient 6 months after an acute spinal cord relapse due to the two lesions between $C 1$ and $C 3$ (yellow circles). $\boldsymbol{b}$, Spectroscopic volume of interest between $C 1$ and $C 3$ on the coronal image. c, Regions of interest located in the white matter columns on the axial diffusivity maps. $\boldsymbol{d}$, FSPGR image at $\mathrm{C2}-\mathrm{C} 3$ with the regions of interest contoured around the cord. $\boldsymbol{e}$, Spectrum obtained by the LCModel analysis of the volume of interest shown in $\boldsymbol{b}$. The NAA concentration was $3.94 \mathrm{~mm} / \mathrm{L}$ (percentage $\mathrm{SD}=11$ ).

ucl.ac.uk/research/medic/camino/pmwiki/pmwiki.php). Three patients' datasets containing four volumes $(6 \%)$ affected by imaging artifacts were excluded from the analysis. Maps of axial diffusivity, which is the principal eigenvalue of the diffusion tensor and represents the preferential direction of water diffusion aligned with the main orientation of axonal fibers, were obtained. The directionality of the principal eigenvalue compared with the spinal cord anatomical structures was visually checked in each subject by displaying the principal eigenvector on the corresponding fractional anisotropy map (Wheeler-Kingshott and Cercignani, 2009). Reduced axial diffusivity reflects axonal degeneration (Kim et al., 2006).

Four regions-of-interest (ROIs) were placed on the b0 images between $\mathrm{C} 1$ and $\mathrm{C} 3$ (on the basis of the slice prescription) in the white matter columns, where white matter tracts are located, and then automatically transferred to axial diffusivity maps (Fig. 1c). The ROIs were carefully placed to minimize partial volume effect originating from the CSF; they included both lesions and normal-appearing tissue, as it was not possible to distinguish the lesional area from the normal-appearing tissue on the b0 maps. For each subject, the mean value of axial diffusivity of the four
ROIs was calculated. In addition, for each subject, the mean axial diffusivity of the whole spinal cord between $\mathrm{C} 1$ and $\mathrm{C} 3$ was also calculated by contouring the spinal cord on b0 images using a semiautomated thresholding technique (Plummer, 1992) and then automatically transferring these regions to axial diffusivity maps.

The final analysis performed was volume-acquired, inversionprepared, fast-spoiled-gradient recalled (FSPGR) sequence $(\mathrm{TR}=13.2$ $\mathrm{ms}$, TE $=4.2 \mathrm{~ms}$, inversion time $=450 \mathrm{~ms}$, flip angle $=20^{\circ}$, FOV $250 \times 250 \mathrm{~mm}^{2}$, matrix $256 \times 256,60$ contiguous sagittal slices, $1 \mathrm{~mm}$ slice thickness). The cord cross-sectional cord area at C2-C3 was calculated using a semiautomated method (Losseff et al., 1996) (Fig. 1d). Reduced cord area in patients when compared with controls suggests ongoing neural degeneration (Miller et al., 2002).

Statistical analysis. Differences in imaging measures [NAA, axial diffusivity of both the spinal cord columns and the entire spinal cord (i.e., white matter and gray matter) between $\mathrm{C} 1$ and $\mathrm{C} 3$, and cord cross-sectional area] between patients and controls were assessed using the Mann-Whitney $U$ test, as these were not normally distributed.

In MS patients, we assumed that NAA concentration is explained by the combination of axonal structural integrity and mitochondrial metabolism. This relationship can be represented in general linear as: $Y=X_{1}+X_{2}+\varepsilon$. $Y$ is the NAA concentration, $X_{1}$ is the axial diffusivity of the spinal cord columns, and $X_{2}$ is the cord cross-sectional area. $\varepsilon$ is the residual variance in NAA concentration (termed ResNAA) after accounting for axial diffusivity and cord cross-sectional area, and should possess information about mitochondrial metabolism. We assumed that all the structural contributions to NAA concentration were captured by these two structural measures, although minor axonal loss not reflected by these in vivo measures cannot be excluded. A similar rationale behind this statistical approach was previously applied with success (looking at functional imaging measures) (Toosy et al., 2005).

Next, to determine the association between ResNAA and clinical disability, an ordinal logistic regression was run using three EDSS categories (EDSS $<3.0$; EDSS between 3.5 and 5.0; EDSS > 5.5) as dependent variables and ResNAA as covariate. For 9HPT, inverse TWT and MSWS12 linear regression analyses were used, entering the clinical score as the dependent variable and ResNAA as covariate. To corroborate findings obtained with this analysis, multiple regression analyses were performed, selecting total NAA as the dependent variable and the clinical scores (i.e., three EDSS categories, 9HPT, TWT, and MSWS12), axial diffusivity in the spinal cord columns, and cord area, as independent variables. This tests whether disability was associated with NAA while adjusting for structural axonal loss, as measured by axial diffusivity in the spinal cord columns and cord area.

Considering that the NAA concentration was measured in a voxel encompassing both gray and white matters, the whole analysis was repeated by specifying a general linear model that included the axial diffusivity of the entire spinal cord (i.e., both white and gray matters) between $\mathrm{C} 1$ and $\mathrm{C} 3$, instead of that confined to the spinal cord columns; this new ResNAA obtained was then associated with clinical scales by using regression analyses, as explained above. Multiple regression analyses were then run to test whether disability scores were associated with NAA while adjusting for axial diffusivity of the entire spinal cord and cord crosssectional area.

Statistical analyses were performed using SPSS 11.5 for Windows. $p$ values $<0.05$ were considered to be significant. 


\section{Results}

Patients showed lower NAA concentration and cross-sectional cord area than controls; there was no difference in axial diffusivity of the spinal cord columns or the whole spine between patients and controls (Table 1 ).

In patients, ResNAA was associated with disability. In particular, patients with higher ResNAA (greater mitochondrial function) were less likely to have higher EDSS (greater disability) [odds ratio $=$ $0.09, p=0.034,95 \%$ confidence intervals $(\mathrm{CIs})=0.01$ and 0.84 ] (Fig. 2a). Lower ResNAA was associated with worse walking ability, as measured by TWT and MSWS12 [inverse TWT: $p=0.024$, parameter estimate $(\mathrm{PE})=0.026,95 \%$ CIs $=0.004,0.05 ;$ MSWS12: $p=0.013$, $\mathrm{PE}=-10.7,95 \%$ CIs $=-18.4,-2.9)$ (Fig. 2b,c).

For the multiple regression models, greater NAA itself was associated with lower EDSS (lower disability) ( $\mathrm{PE}=$ $-2.063, p=0.012$, 95\% CIs -3.49, -0.64 ) and better walking ability (inverse TWT: $\mathrm{PE}=50.1, p=0.031,95 \%$ CIs 6.5 , 93.7; MSWS12: $\mathrm{PE}=-0.14, p=0.012$, $95 \%$ CIs $-0.24,-0.04)$. NAA was not associated with axial diffusivity of the spinal cord columns (EDSS: $\mathrm{PE}=0.002, p=0.5$; TWT: $\mathrm{PE}=0.004, p=$ 0.2 ; MSWS12: $\mathrm{PE}=0.005, p=0.1)$ or cord cross-sectional area (EDSS: $\mathrm{PE}=0.03, p=0.7$; TWT: $\mathrm{PE}=0.09, p=0.3$; MSWS12: $\mathrm{PE}=0.09, p=0.2$ ). This suggests that the relationship between NAA and clinical disability is preserved after simultaneously adjusting for the structural components of NAA and so is very likely to be determined by its metabolic component.

When the analysis was repeated using the mean axial diffusivity of the whole spinal cord $(\mathrm{C} 1-\mathrm{C} 3)$ instead of the spinal cord columns, similar significant results were obtained; in particular, lower ResNAA was associated with greater disability, as measured by EDSS (odd ratio $=0.13, p=0.039,95 \%$ CIs 0.02,0.9), TWT $(p=0.013, \mathrm{PE}=0.025,95 \%$ CIs $0.007,0.04)$ and MSWS12 $(p=$ $0.008, \mathrm{PE}=-10.1,95 \% \mathrm{CIs}-16.8,-3.4)$. In addition, greater NAA itself was associated with lower disability, as measured by EDSS (PE $=-1.95, p=0.009,95 \%$ CIs $-3.24,-0.66)$, TWT $(\mathrm{PE}=51.1, p=0.015,95 \%$ CIs 13.5, 88.7), and MSWS12 $(\mathrm{PE}=$ $-0.14, p=0.007,95 \%$ CIs $-0.23,-0.05)$ when adjusted for axial diffusivity of the entire spinal cord and cord cross-sectional area, which were not significantly associated with NAA (PEs and $p$ values for axial diffusivity in the entire spinal cord in the models with EDSS, TWT, and MSWS12 were as follows: 0.001, $p=0.6$; $0.006, p=0.1$, and $0.006, p=0.07$, respectively. PEs and $p$ values for cord cross-sectional area in the models with EDSS, TWT, and MSWS12 were as follows: $0.02, p=0.8 ; 0.11, p=0.1$, and $0.1, p=$ 0.2 , respectively).

\section{Discussion}

Our findings show that this novel approach is clinically relevant, and can be used to estimate in vivo the component of NAA, which is unexplained by structural measures and, therefore, may reflect mitochondrial metabolism; and to assess its relative contribution to disability. This method may be extended to other disorders and
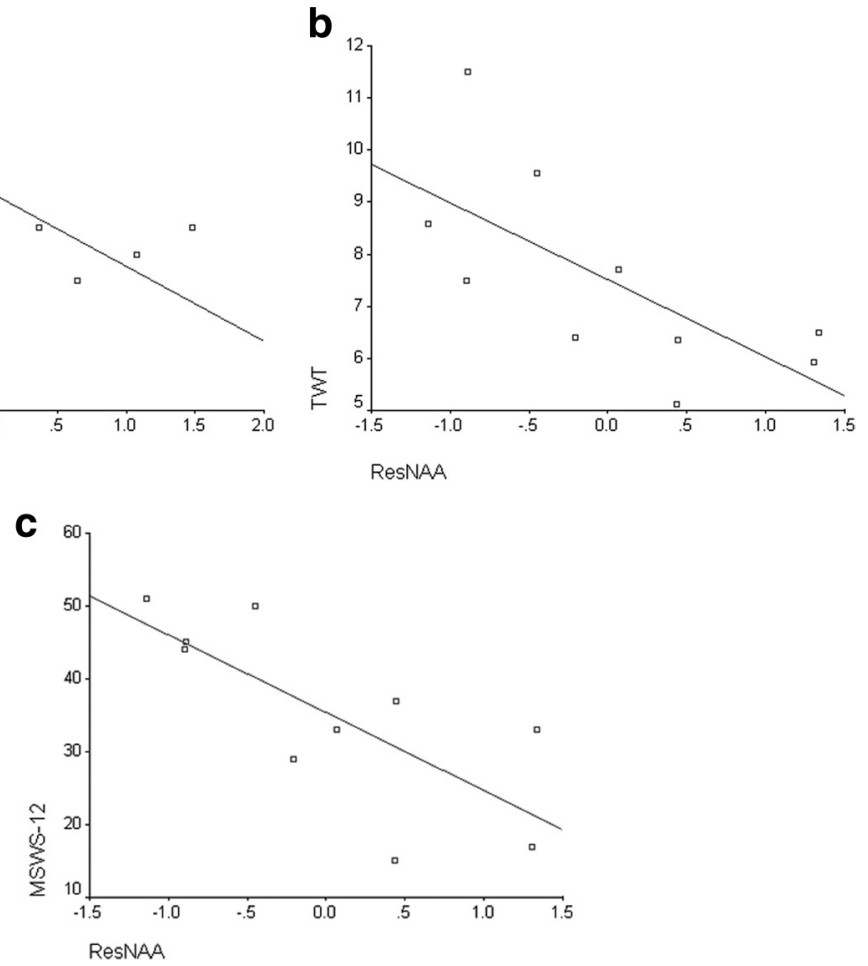

account structural imaging meaFigure 2. Relationship between the residual variance in NAA concentration after taking into account structural imaging mea-
sures and clinical scores. $\boldsymbol{a}$, Scatter plot of ResNAA versus EDSS $\left(R^{2}=0.5\right)$ with a fitted line. $\boldsymbol{b}$, Scatter plot of ResNAA versus TWT $\left(R^{2}=0.42\right)$.c, Scatter plot of ResNAA versus MSWS12 $\left(R^{2}=0.4\right)$.

areas of the CNS and include other structural imaging measures, as long as they are calculated in the same region. Hence, it has great potential. There are, however, several considerations. Although more complicated models can be used to estimate the mitochondrial metabolic component of NAA, we started with a simple model and used parameters that were chosen a priori. Our analysis assumes that the residual variance within the patient group in NAA represents mitochondrial metabolism after taking into account the structural contributions of axial diffusivity, as measured in the spinal cord columns, the entire spinal cord, and cross-sectional cord area, which were chosen a priori. However, ResNAA may be influenced by some noise, which is inevitable in imaging studies. This may predispose to type II rather than type I errors. Furthermore, axial diffusivity was not significantly different between patients and controls. Therefore, we conducted a post hoc analysis that reevaluated ResNAA with cord crosssectional area only. We found that the correlations between the resulting ResNAA and disability remained significant (data not shown), suggesting that, in this particular patient cohort, axial diffusivity did not contribute to the model.

The ROIs used to estimate NAA and axial diffusivity included both lesions and normal-appearing tissue, potentially subjecting them to partial volume confounds. Despite this, and the relatively small cohort, which, however, was studied during the remission phase following an acute relapse, significant effects were still observed, suggesting that this technique is sensitive at extracting mitochondrial metabolism contributions. Also, mitochondrial dysfunction in MS is probably not confined to lesions but also present in normal-appearing tissue (Mahad et al., 2008). Therefore, partial volume effects may not dilute the NAA metrics as much as anticipated. Further technical developments and the use of high magnetic fields will increase spectral spatial and temporal resolution and signal-to-noise ratio. The ROI approach used for 
the analysis of diffusion maps allowed us to calculate the axial diffusivity within the white matter cord columns, i.e., in regions of high fiber coherence. In addition, we repeated the whole analysis by calculating the axial diffusivity in the whole spinal cord from $\mathrm{C} 1$ to $\mathrm{C} 3$ (i.e., both white and gray matters), and significant findings were confirmed.

With regard to the association between mitochondrial function and disability, we found that the residual variance in NAA concentration, after accounting for structural measures (axial diffusivity and cord cross-sectional area), is significantly associated with clinical impairment (EDSS, TWT, and MSWS12) in MS patients after a cervical cord relapse. This is independent of measures of structural integrity, which do not correlate with disability, supporting the hypothesis that mitochondrial dysfunction plays an important role in contributing to disability in MS. Our additional analysis shows that NAA concentration is associated with disability while adjusting for structural measures, suggesting that the relationship between NAA and disability is not explained by axonal loss, but possibly by the metabolic component of NAA.

Future studies will explore the role of mitochondrial dysfunction, estimated indirectly by combining imaging measures in strategic brain regions, in determining disability in MS. We focused on the spinal cord because this region plays a key role in causing clinical impairment, and imaging measures of neurodegeneration in this area are robust. We have previously shown that, in the same group of patients, there was a sustained increase in the spinal cord NAA concentration between 1 and 6 months, which was concurrent with the development of cord atrophy and correlated with clinical recovery (Ciccarelli et al., 2010), in agreement with previous brain studies (De Stefano et al., 1995). This suggested that increased mitochondrial metabolism is a compensatory repair mechanism.

In conclusion, the importance of the metabolic component of the NAA will need to be further investigated in view of possible applications to trials that assess agents targeting mitochondrial activity to prevent axonal loss.

\section{References}

Bates TE, Strangward M, Keelan J, Davey GP, Munro PM, Clark JB (1996) Inhibition of $N$-acetylaspartate production: implications for $1 \mathrm{H}$ MRS studies in vivo. Neuroreport 7:1397-1400.

Bjartmar C, Kidd G, Mörk S, Rudick R, Trapp BD (2000) Neurological disability correlates with spinal cord axonal loss and reduced $\mathrm{N}$-acetyl aspartate in chronic multiple sclerosis patients. Ann Neurol 48:893-901.

Chang LC, Jones DK, Pierpaoli C (2005) RESTORE: robust estimation of tensors by outlier rejection. Magn Reson Med 53:1088-1095.

Ciccarelli O, Wheeler-Kingshott CA, McLean MA, Cercignani M, Wimpey K, Miller DH, Thompson AJ (2007) Spinal cord spectroscopy and diffusion-based tractography to assess acute disability in multiple sclerosis. Brain 130:2220-2231.
Ciccarelli O, Altmann DR, McLean MA, Wheeler-Kingshott CA, Wimpey K, Miller DH, Thompson AJ (2010) Spinal cord repair in MS: does mitochondrial metabolism play a role? Neurology 74:721-727.

Cutter GR, Baier ML, Rudick RA, Cookfair DL, Fischer JS, Petkau J, Syndulko K, Weinshenker BG, Antel JP, Confavreux C, Ellison GW, Lublin F, Miller AE, Rao SM, Reingold S, Thompson A, Willoughby E (1999) Development of a multiple sclerosis functional composite as a clinical trial outcome measure. Brain 122:871-882.

De Stefano N, Matthews PM, Antel JP, Preul M, Francis G, Arnold DL (1995) Chemical pathology of acute demyelinating lesions and its correlation with disability. Ann Neurol 38:901-909.

Dowell NG, Jenkins TM, Ciccarelli O, Miller DH, Wheeler-Kingshott CA (2009) Contiguous-slice zonally oblique multislice (CO-ZOOM) diffusion tensor imaging: examples of in vivo spinal cord and optic nerve applications. J Magn Reson Imaging 29:454-460.

Dutta R, McDonough J, Yin X, Peterson J, Chang A, Torres T, Gudz T, Macklin WB, Lewis DA, Fox RJ, Rudick R, Mirnics K, Trapp BD (2006) Mitochondrial dysfunction as a cause of axonal degeneration in multiple sclerosis patients. Ann Neurol 59:478-489.

Fischer JS, Jak AJ, Kniker JE, Rudick RA, Cutter G (1999) Administration and scoring manual for the multiple sclerosis functional composite meaure (MSFC). New York: Demos.

Hobart JC, Riazi A, Lamping DL, Fitzpatrick R, Thompson AJ (2003) Measuring the impact of MS on walking ability: the 12-Item MS Walking Scale (MSWS-12). Neurology 60:31-36.

Kim JH, Budde MD, Liang HF, Klein RS, Russell JH, Cross AH, Song SK (2006) Detecting axon damage in spinal cord from a mouse model of multiple sclerosis. Neurobiol Dis 21:626-632.

Kurtzke JF (1983) Rating neurologic impairment in multiple sclerosis: an expanded disability status scale (EDSS). Neurology 33:1444-1452.

Losseff NA, Webb SL, O'Riordan JI, Page R, Wang L, Barker GJ, Tofts PS, McDonald WI, Miller DH, Thompson AJ (1996) Spinal cord atrophy and disability in multiple sclerosis: a new reproducible and sensitive MRI method with potential to monitor disease progression. Brain 119:701708 .

Mahad D, Lassmann H, Turnbull D (2008) Review: mitochondria and disease progression in multiple sclerosis. Neuropathol Appl Neurobiol 34:577-589.

McDonald WI, Compston A, Edan G, Goodkin D, Hartung HP, Lublin FD, McFarland HF, Paty DW, Polman CH, Reingold SC, Sandberg-Wollheim M, Sibley W, Thompson A, van den Noort S, Weinshenker BY, Wolinsky JS (2001) Recommended diagnostic criteria for multiple sclerosis: guidelines from the international panel on the diagnosis of multiple sclerosis. Ann Neurol 50:121-127.

Miller DH, Barkhof F, Frank JA, Parker GJ, Thompson AJ (2002) Measurement of atrophy in multiple sclerosis: pathological basis, methodological aspects and clinical relevance. Brain 125:1676-1695.

Plummer DL (1992) Dispimage: a display and analysis tool for medical images. Rev Neuroradiol 5:489-495.

Toosy AT, Hickman SJ, Miszkiel KA, Jones SJ, Plant GT, Altmann DR, Barker GJ, Miller DH, Thompson AJ (2005) Adaptive cortical plasticity in higher visual areas after acute optic neuritis. Ann Neurol 57:622-633.

Wheeler-Kingshott CA, Cercignani M (2009) About "axial" and "radial" diffusivities. Magn Reson Med 61:1255-1260. 